\title{
The effect of boron doping on crystal structure, magnetic properties and magnetocaloric
}

\section{effect of DyCo2}

C. L. Wang, ${ }^{1,2, a)}$ J. Liu, ${ }^{1,3}$ Y. Mudryk ${ }^{1}$, K. A. Gschneidner, Jr., ${ }^{1,3}$ Y. Long, ${ }^{2}$ V. K. Pecharsky ${ }^{1,3}$

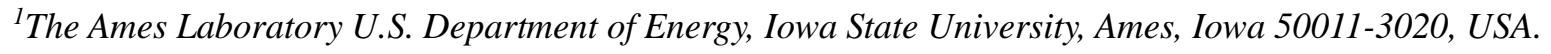

${ }^{2}$ School of Materials Science and Engineering, University of Science and Technology of Beijing, 100083, People's Republic of China.

${ }^{3}$ Department of Materials Science and Engineering, Iowa State University, Ames, Iowa 50011-2300, USA.

The magnetic properties and magnetic entropy changes of $\mathrm{DyCo}_{2} \mathrm{~B}_{\mathrm{x}}(\mathrm{x}=0,0.05,0.1,0.2)$ alloys were investigated. The Curie temperature $\left(\mathrm{T}_{\mathrm{C}}\right)$ increases with increasing $\mathrm{B}$ concentration. The frequency dependence of ac magnetic susceptibility of $\mathrm{DyCo}_{2}$ caused by the narrow domain wall pinning effect is depressed by B doping, but the coercivity and the magnetic viscosity are prominently increased in the $\mathrm{B}$ doped alloys. The magnetic transition nature of $\mathrm{DyCo}_{2} \mathrm{~B}_{\mathrm{x}}$ changes from the first-order to the second-order with increasing $\mathrm{x}$, which leads to the decrease of the maximum magnetic entropy change. However, the relative cooling power ( $\mathrm{RCP}$ ) of $\mathrm{DyCO}_{2}$ and the B doped alloys remains nearly constant.

a) Address: No. 30 Xueyuan Road, Haidian District, Beijing 100083, People's Republic of China. Electronic mail:wangchaolun2004@163.com. 


\section{INTRODUCTION}

The Laves phases $\mathrm{RM}_{2}(\mathrm{R}$ is a rare earth element and $\mathrm{M}$ is a transition metal) keep attracting a lot of research attention, because of their rich magnetic properties, such as giant magnetoresistance, magnetostriction, and magnetocaloric effects. ${ }^{1-4}$ A particular property of the $\mathrm{DyCo}_{2}$ compound is the narrow domain wall pinning effect manifested by the irreversibility of ZFC and FCC magnetization, and the ZFC peak just below the transition temperature, ${ }^{5}$ which is also a signature of the spin glass system. The narrow domain wall pinning effect occurs due to the high magnetocrystalline anisotropy of $\mathrm{DyCo}_{2},{ }^{6,7}$ and this effect depends strongly upon the applied magnetic field strength. ${ }^{5}$ For the narrow domain wall systems, there is an energy difference between the center of the wall located at an atomic plane and the one that is located between two planes. Such difference blocks the domain wall motion and leads to the domain wall pinning effect. ${ }^{8}$ Compared to the domain wall pinning caused by the impurities and defects, the narrow domain wall pinning is an intrinsic property in $\mathrm{DyCo} 2$.

Magnetic refrigeration is a promising technology, which can replace the traditional vapor-compression refrigeration due to the higher efficiency and eco-friendliness. Since the discovery of the giant magnetocaloric effect (GMCE) in the intermetallic compound $\mathrm{Gd}_{5} \mathrm{Si}_{2} \mathrm{Ge}_{2},{ }^{9}$ increasing attention has been devoted to GMCE near room-temperature resulting in the discovery of other promising GMCE materials such as $\mathrm{La}\left(\mathrm{Fe}_{1-\mathrm{x}} \mathrm{Si}_{\mathrm{x}}\right)_{13}$ and its hydrides, $\mathrm{MnFeP}_{1-\mathrm{x}} \mathrm{As} \mathrm{s}_{\mathrm{x}}, \mathrm{MnAs}_{1-\mathrm{x}} \mathrm{Sb}_{\mathrm{x}}$, Heusler alloys (e.g. $\mathrm{Ni}_{51.5} \mathrm{Mn}_{22.7} \mathrm{Ga}_{25.8}$ ), $\mathrm{RM}_{2}$ Laves phases and others. ${ }^{10-16}$

The Laves phase $\mathrm{DyCo}_{2}$ has the highest first-order magnetic transition (FOMT) temperature (140 K) in the $\mathrm{RCo}_{2}$ family of alloys, but it is still far below the room temperature. To increase the transition temperature of $\mathrm{DyCo}_{2}$, element substitutions have been extensively studied over the past few years. For example, partial replacement of Dy by Gd and $\mathrm{Tb},{ }^{17,18}$ and substitution of Co by $\mathrm{Ga}, \mathrm{Fe}, \mathrm{Al}$, and 
$\mathrm{Si}^{6,19-21}$ were investigated. All the results show that the Curie temperature rises with the increase of lattice constant. However, the order of the magnetic transition changes from first to second. The non-monotonic increase of $\mathrm{T}_{\mathrm{C}}$ in case of $\mathrm{Si}$ substitution was explained by the competition among magnetovolume effect and magnetic dilution effect. ${ }^{6}$ Moreover, the study of the pressure effects on $\mathrm{DyCo}_{2}$ reveals that the Curie temperature decreases with the application of external pressure due to the contraction of the lattice parameter. ${ }^{22}$ Doping by interstitial elements (H, C, and B) usually leads to an increase of the lattice constant, and the $\mathrm{H}$ absorption has been successfully applied to adjust the Curie temperature of $\mathrm{La}\left(\mathrm{Fe}_{0.89} \mathrm{Si}_{0.11}\right)_{13}$ from $190 \mathrm{~K}$ to room-temperature, while preserving the first-order of the magnetic transition. ${ }^{15}$ However, the hydrogenation treatment for $\mathrm{DyCo}_{2}$ leads to disproportionation and amorphization of the compound. ${ }^{23}$

In this paper, the magnetocaloric effect and magnetic properties (such as ac susceptibilities, magnetic hysteresis, magnetic relaxation and magnetic phase transition at Curie temperature) of $\mathrm{DyCo}_{2} \mathrm{~B}_{\mathrm{x}}(\mathrm{x}=0,0.05,0.1,0.2)$ alloys were studied, due to the influences of interstitial element $\mathrm{B}$ on the exchange interaction and the domain wall mobility of $\mathrm{DyCo}_{2}$.

\section{П. EXPERIMENTAL DETAILS}

The $\mathrm{DyCo}_{2} \mathrm{~B}_{\mathrm{x}}(\mathrm{x}=0,0.05,0.1,0.2)$ alloys were prepared by arc-melting of the constituents in an argon atmosphere. The alloys were turned over several times to insure the homogeneity of the sample. The Dy metal was prepared by the Materials Preparation Center ${ }^{24}$ of the Ames Laboratory and it was 99.8 at.\% (99.97 wt.\%) pure with the major impurities, given as at. ppm (wt. ppm), as follows: O-802(79), C- 1271(94), N-104(9), Hf-109(120). The Co metal of 99.95 wt.\% purity was purchased from Alfa Aesar Inc. The purity of B was 99.5 wt.\%. The arc-melted ingots were annealed in evacuated silica tubes at $1173 \mathrm{~K}$ for 6 days, and then quenched in ice-water slurry. 
Structural and phase analyses were performed by collecting and analyzing the powder x-ray diffraction (XRD) patterns obtained at room temperature on PANalytical X'Pert Pro diffractometer using $\mathrm{Cu} \mathrm{K} \alpha_{1}$ radiation. The lattice parameters of the obtained phases and the concentration of the minor phase (when present) were calculated by the Rietveld method using the LHPM Rietica software. ${ }^{25}$ Magnetic properties of bulk polycrystalline samples were measured by superconducting quantum interference device (SQUID) magnetometer, MPMS-XL7 from Quantum Design Inc. The ZFC, FCC and FCW magnetization measurements were made as follows: the ZFC curve was measured by cooling the sample down to $2 \mathrm{~K}$ without magnetic field, then a magnetic field of 100 Oe was applied, and the magnetization of a sample was measured during heating form $2 \mathrm{~K}$ to $200 \mathrm{~K}$, with the temperature rate of $2 \mathrm{~K} / \mathrm{min}$. The FCC curve was measured by sweeping down the temperature from $200 \mathrm{~K}$ to $2 \mathrm{~K}$ in applied field of 100 Oe. The field-cooled-warming (FCW) curve was obtained by cooling the sample down in presence of 100 Oe magnetic field, then measuring the magnetization from $2 \mathrm{~K}$ to $200 \mathrm{~K}$ under magnetic field of $100 \mathrm{Oe}$.

\section{Results and discussion}

\section{A. XRD and magnetization measurements}

The XRD patterns of $\mathrm{DyCo}_{2} \mathrm{~B}_{\mathrm{x}}(\mathrm{x}=0,0.05,0.1,0.2)$ alloys containing the $\mathrm{C} 15\left(\mathrm{MgCu}_{2}\right.$-type structure) Laves phase as a main phase are shown in Fig. 1. The impurity phase $\mathrm{DyCo}_{2} \mathrm{~B}_{2}$ ( $\mathrm{BaAl}_{4}$-type) was found in $\mathrm{DyCo}_{2} \mathrm{~B}_{0.2}$ and its Bragg peaks are marked by black diamonds; the concentration of this phase is about $8 \mathrm{wt} . \%$ as determined by Rietveld refinement. 


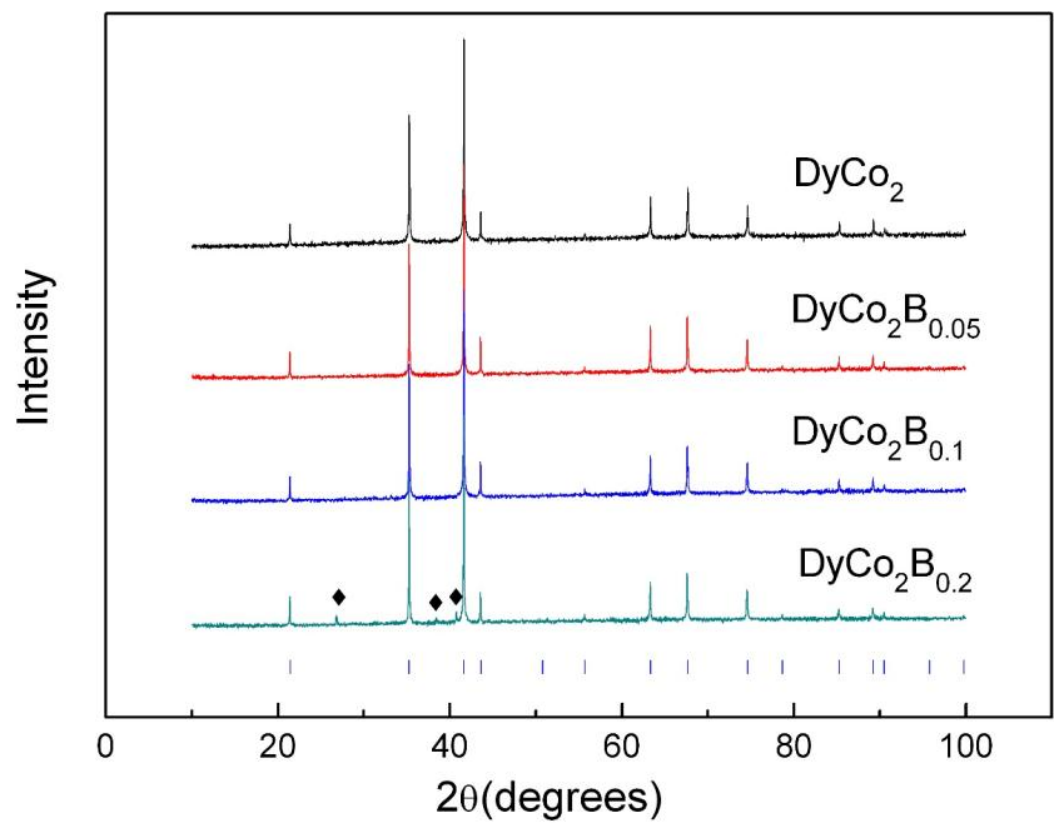

FIG. 1. XRD patterns of $\mathrm{DyCo}_{2} \mathrm{~B}_{\mathrm{x}}(\mathrm{x}=0,0.05,0.1,0.2)$, vertical bars indicate the calculated positions of the Bragg peaks of the $\mathrm{MgCu}_{2}$-type Laves phase. Diamonds on the $\mathrm{DyCo}_{2} \mathrm{~B}_{0.2}$ alloy signify the impurity phase $\mathrm{DyCo}_{2} \mathrm{~B}_{2}\left(\mathrm{BaAl}_{4}\right.$-type).

The lattice parameters of $\mathrm{DyCo}_{2} \mathrm{~B}_{\mathrm{x}}(\mathrm{x}=0,0.05,0.1,0.2)$ calculated by the Rietveld refinement are listed in Table 1. Due to B doping, the lattice parameter expands from 7.191(1) $\AA$ ( $\left.\mathrm{DyCo}_{2}\right)$ to 7.195(1) $\mathrm{A}\left(\mathrm{DyCo}_{2} \mathrm{~B}_{0.1}\right)$. However, the lattice parameter of $\mathrm{DyCo}_{2} \mathrm{~B}_{0.2}$ and $\mathrm{DyCo}_{2} \mathrm{~B}_{0.1}$ is the same. This indicates that the $\mathrm{DyCo}_{2} \mathrm{~B}_{\mathrm{x}}$ solid solution terminates near $x=0.1$. For $\mathrm{AB}_{2}(\mathrm{C} 15$-type $)$ Laves phase, there are three types of tetrahedral sites available which are presented in the following format, atom type + atom number (Wyckoff sites): B4(8b), AB3(32e) and A2B2(96g), and interstitial atoms prefer $\mathrm{AB} 3$ and $\mathrm{A} 2 \mathrm{~B} 2$ sites. ${ }^{26,27}$ Therefore, those two positions are potential interstitial sites for boron in $\mathrm{DyCo}_{2} \mathrm{~B}_{\mathrm{x}}$ compounds. Distortions caused by boron atoms may account for the small solid solubility in DyCo2.

As shown in Fig. 2, below $\mathrm{T}_{\mathrm{C}}$ noticeable thermomagnetic irreversibility between $\mathrm{ZFC}$ and FCC magnetizations occurs in 100 Oe magnetic field in $\mathrm{DyCo}_{2}$ and is attributed to the narrow domain 
wall pinning effect. ${ }^{8,28}$ A weak anomaly was found in ZFC curve at $20 \mathrm{~K}$ (labeled as $\mathrm{T}_{\mathrm{a}}$, see Fig. 2a). On the further increase of temperature ZFC magnetization forms a broad peak below $\mathrm{T}_{\mathrm{C}}(136(1) \mathrm{K})$, and then drops sharply at the Curie temperature showing a magnetic transition from the ferrimagnetic state ${ }^{4}$ to the paramagnetic state. The field cooling process stabilizes the magnetic moments along the magnetic field direction. As a result, both the FCC and FCW curves have a stable magnetization below $\mathrm{T}_{\mathrm{C}}$. An obvious irreversibility of FCC and FCW curves below $\mathrm{T}_{\mathrm{C}}$ (Fig. 2a) is often observed in materials that show a significant time dependence of remanent magnetization ${ }^{29}$ which will be discussed in part B.

The dc magnetization curves of $\mathrm{DyCo}_{2} \mathrm{~B}_{\mathrm{x}}(\mathrm{x}=0.05$ and 0.1$)$ displayed in Fig. 2(b) and (c) are similar. Because of the enhancement of domain pinning effect by interstitial B atoms, the ZFC magnetizations are close to zero below $43 \mathrm{~K}\left(\mathrm{~T}_{\mathrm{P}}\right.$, indicated by arrows in Figs. $\left.2 \mathrm{~b}, \mathrm{c}\right)$. Above $\mathrm{T}_{\mathrm{P}}$ the ZFC magnetization increases quickly and reaches maximum just below $\mathrm{T}_{\mathrm{C}}$. The thermomagnetic irreversibility between FCC and FCW curves below $\mathrm{T}_{\mathrm{C}}$ exists in both of the doped samples.

The Curie temperatures of $\mathrm{DyCo}_{2} \mathrm{~B}_{\mathrm{x}}(\mathrm{x}=0,0.05,0.1,0.2)$ are increased from $136(1) \mathrm{K}(\mathrm{x}=0)$ to 147(1) $\mathrm{K}(\mathrm{x}=0.2)$ as listed in Table 1 . The increase of $\mathrm{T}_{\mathrm{C}}$ is a result of the volume expansion which leads to the $3 d$ band narrowing. ${ }^{6}$ 

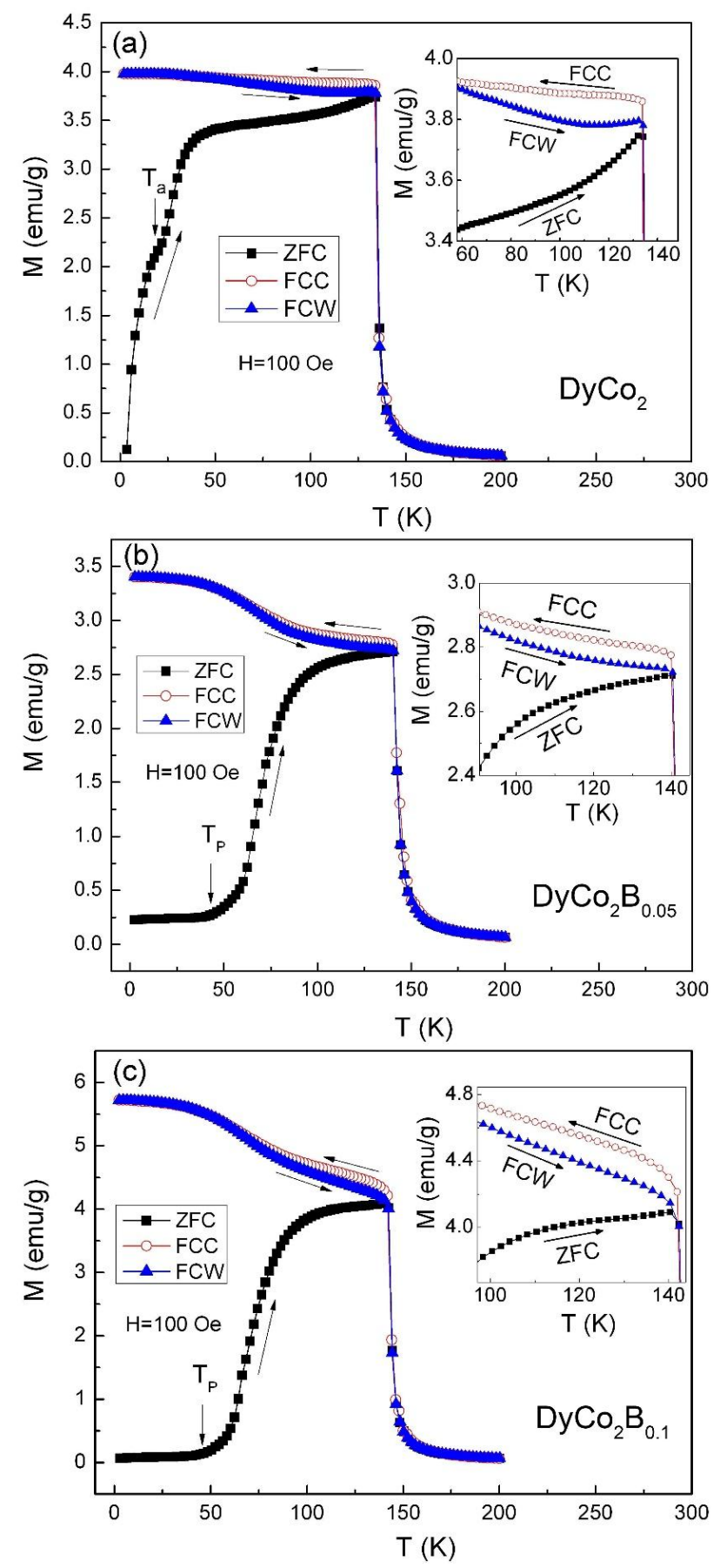

FIG. 2. The ZFC-FCC-FCW magnetization of $\mathrm{DyCo}_{2} \mathrm{~B}_{\mathrm{x}}(\mathrm{x}=0,0.05,0.1)$ measured in a 100 Oe magnetic field. The insets show a detailed view of dc magnetization curves near the Curie temperature.

The inset of Fig. 3 shows that the paramagnetic susceptibilities of $\mathrm{DyCo}_{2} \mathrm{~B}_{\mathrm{x}}$ follow the Curie-Weiss law: 


$$
H / M=N p_{e f f}^{2} / 3 k\left(T-\theta_{p}\right)
$$

where $H$ is the applied magnetic field, $M$ is the molar magnetization, $N$ is Avogadro's number, $p_{\text {eff }}$ is the effective magnetic moment, $k$ is Boltzmann's constant, $T$ is the absolute temperature, and $\theta_{p}$ is the paramagnetic Weiss temperature. The calculated effective magnetic moment per formula unit $p_{\text {eff }}$ and the paramagnetic Weiss temperature $\theta_{p}$ are listed in the Table 1 . Similar to $\mathrm{T}_{\mathrm{C}}, \theta_{p}$ of $\mathrm{DyCo}_{2} \mathrm{~B}_{\mathrm{x}}$ increases from $133(1) \mathrm{K}(\mathrm{x}=0)$ to $142(1) \mathrm{K}(\mathrm{x}=0.1)$, however, the $p_{\text {eff }}$ decreases from $9.56 \mu_{\mathrm{B}} / \mathrm{f} . \mathrm{u}$. $(\mathrm{x}=0)$ to $9.23 \mu_{\mathrm{B}} / \mathrm{f} . \mathrm{u}$. $(\mathrm{x}=0.1)$. The increase of paramagnetic Weiss temperature with boron concentration also results from the volume expansion which enhances the exchange interaction of Co sublattice. It is important to note that no short range order effects (such as parimagnetic effect ${ }^{30}$ ) are observed in the $\mathrm{DyCo}_{2}$ compound prepared using the high-purity rare earth metal.

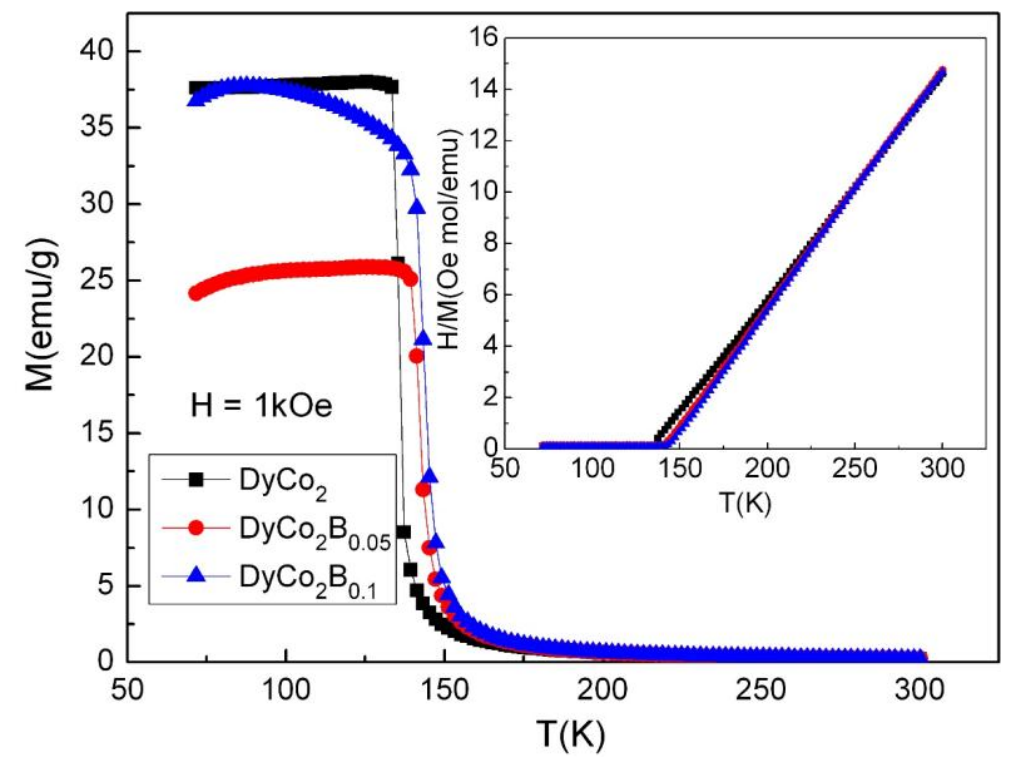

FIG. 3. Temperature dependence of dc magnetization of $\mathrm{DyCo}_{2} \mathrm{~B}_{\mathrm{x}}(\mathrm{x}=0,0.05,0.1)$ measured in $1 \mathrm{kOe}$ field under ZFC condition. The inset shows the inverse susceptibility for the same samples.

The effect of boron on magnetization of $\mathrm{DyCo}_{2}$ is also examined by saturation magnetization measurement at $5 \mathrm{~K}$. By linear fitting the magnetization versus reciprocal magnetic field (not shown here), the saturation magnetizations of $\mathrm{DyCo}_{2} \mathrm{~B}_{\mathrm{x}}(\mathrm{x}=0,0.05,0.1$ and 0.2$)$ are estimated as follow: 
151, 160, 139 and $132 \mathrm{emu} / \mathrm{g}$. The non-monotonic saturation magnetizations of $\mathrm{DyCo}_{2} \mathrm{~B}_{\mathrm{x}}$ alloys may result from the competition between lattice expansion and hybridization effect ${ }^{[31]}$.

The influence of $\mathrm{B}$ doping on the ac susceptibility of $\mathrm{DyCo}_{2} \mathrm{~B}_{\mathrm{x}}$ compounds is studied. The samples were cooled down to $2 \mathrm{~K}$, and then the ac susceptibility was measured from $1 \mathrm{~Hz}$ to 1000 $\mathrm{Hz}$ during heating in zero dc magnetic field (Fig. 4). At low temperatures the $\chi^{\prime}$ and $\chi^{\prime \prime}$ of $\mathrm{DyCo}_{2}$ are both zero due to the domain wall pinning effect, since the 5 Oe ac magnetic field is too small to move the domain walls. With temperature increasing to $\sim 30 \mathrm{~K}$, both real and imaginary parts of ac magnetization begin to increase showing strong frequency dependence. The $\chi^{\prime}$ reaches maximum just below $\mathrm{T}_{\mathrm{C}}$, but the peaks of $\chi^{\prime \prime}$ are at about half of the $\mathrm{T}_{\mathrm{C}}$.

The imaginary susceptibility of $\mathrm{DyCo}_{2}$ has two peaks, the broad peak around $50 \mathrm{~K}$ that shifts to higher temperature with increasing the frequency, and the sharp peak at $\mathrm{T}_{\mathrm{C}}$, which is frequency independent and small. The nonzero imaginary susceptibility indicates a dissipative process caused by the narrow domain wall pinning. When the temperature is high enough (in this case close to $T_{\mathrm{C}}$ ) the domain walls become unrestricted, so the $\chi^{\prime \prime}$ drops to zero. At these temperatures the frequency dependence of $\chi^{\prime}$ disappears too. Spin glass systems also show frequency dependence of ac susceptibility, but the frequency dependence of freezing temperature (defined as the peak of ac susceptibility ${ }^{32}$ ), which is a typical characteristic of a spin glass system does not occur in DyCo 2 .

The frequency dependence of the B doped samples is weaker than that of DyCo2. This may be due to the fact that the frequency dependence of ac susceptibility mainly results from the narrow domain wall pinning effect, which becomes weaker with the temperature increase, as shown by the $\chi^{\prime}$ of DyCo2. Because the doping by $\mathrm{B}$ increases pinning, the domain walls cannot respond the ac magnetic field until temperature reaches $\sim 80 \mathrm{~K}$, where the thermal energy of the system becomes high enough to suppress the pinning and the frequency effects associated with it. In addition, the 
sharp peak of $\chi^{\prime \prime}$ at $\mathrm{T}_{\mathrm{C}}$ disappears, but weak anomaly is still observed.
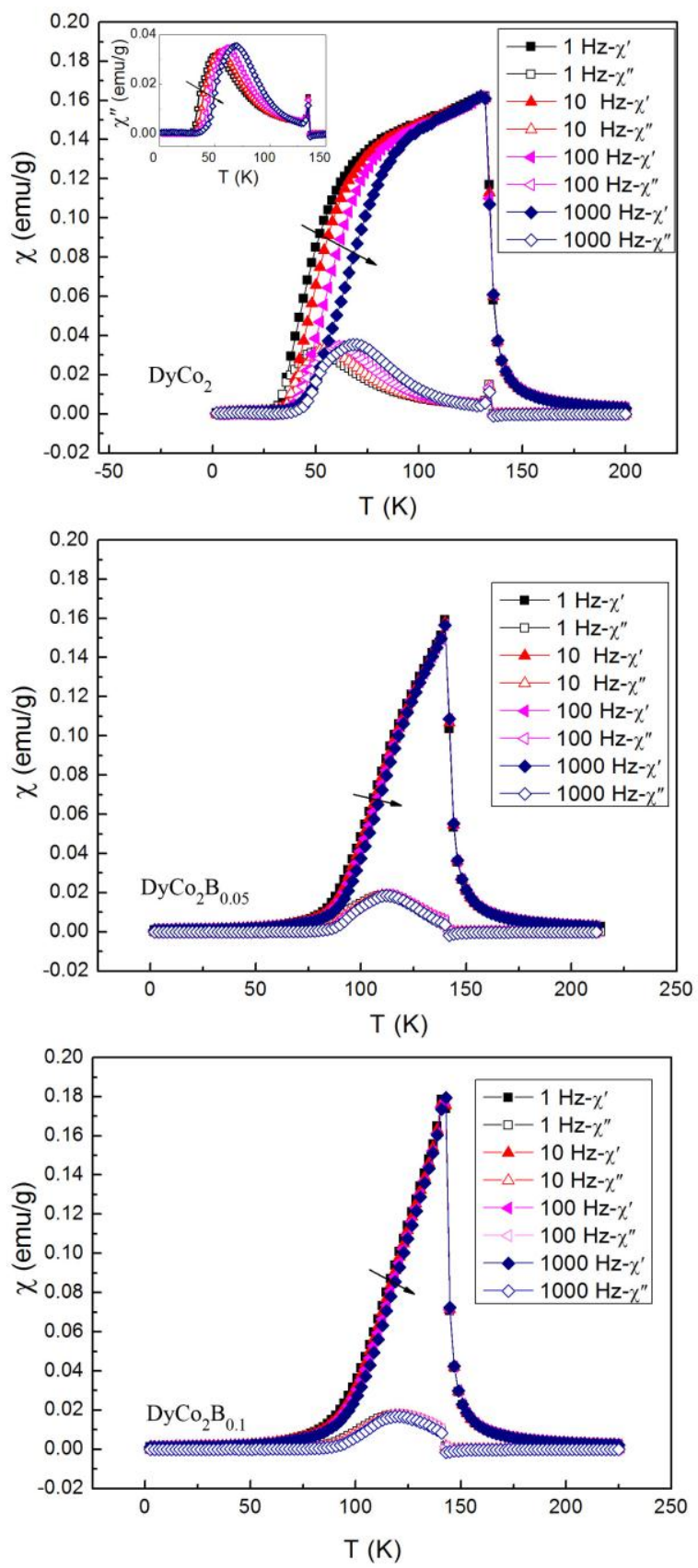

FIG. 4. The temperature dependence of ac susceptibility of $\mathrm{DyCo}_{2} \mathrm{~B}_{\mathrm{x}}(\mathrm{x}=0,0.05,0.1)$ measured in 5

Oe ac field with frequency ranging from $1 \mathrm{~Hz}$ to $1000 \mathrm{~Hz} . \chi^{\prime}$ is the real part (solid symbols) and $\chi^{\prime \prime}$ is the imaginary part (open symbols). The inset of $\mathrm{DyCo}_{2}$ is the amplification of its imaginary susceptibility.

The domain wall pinning effect has an influence on the coercivity determined by measuring the 
magnetic hysteresis loop. Hysteresis loops of $\mathrm{DyCo}_{2} \mathrm{~B}_{\mathrm{x}}$ were performed with magnetic field step of 200 Oe and are shown in Fig. 5. The coercivities of $\mathrm{DyCo}_{2} \mathrm{~B}_{\mathrm{x}}(\mathrm{x}=0,0.05,0.1)$ samples are 0.28(1), 3.29(1) and 4.41(1) kOe respectively, as listed in Table 1. It is possible that the coercivity is increased due to stronger domain wall pinning effect caused by the introduction of the interstitial B atoms (see above). However, modification of the microstructure, namely the precipitation of the secondary phase at the phase boundaries (even though the phase is not seen in the XRD patterns) may also be responsible for the larger coercivity.

For the B doped samples, one can define a critical field called propagation field $\left(H_{p}\right)$, indicated by arrows in Fig. 5, the initial field at which the magnetization starts rising ${ }^{33}$. The propagation fields of $\mathrm{DyCo}_{2} \mathrm{~B}_{\mathrm{x}}(\mathrm{x}=0,0.05,0.1)$ increase with $\mathrm{x}$ from zero to $1.9 \mathrm{kOe}$ and $2.5 \mathrm{kOe}$, respectively. This indicates the enhanced stability of the domain walls. Moreover, all of the samples have unusual initial magnetization curves, which go outside of the hysteresis loop at low magnetic field and then cross the loop back inside when the magnetization is nearly saturated. This abnormal region corresponds to the irreversible domain wall motion and rotation, which imply that the domain wall 'feels' higher resistance during the first time passing though the irreversible domain wall movement region, than that of the second time. 

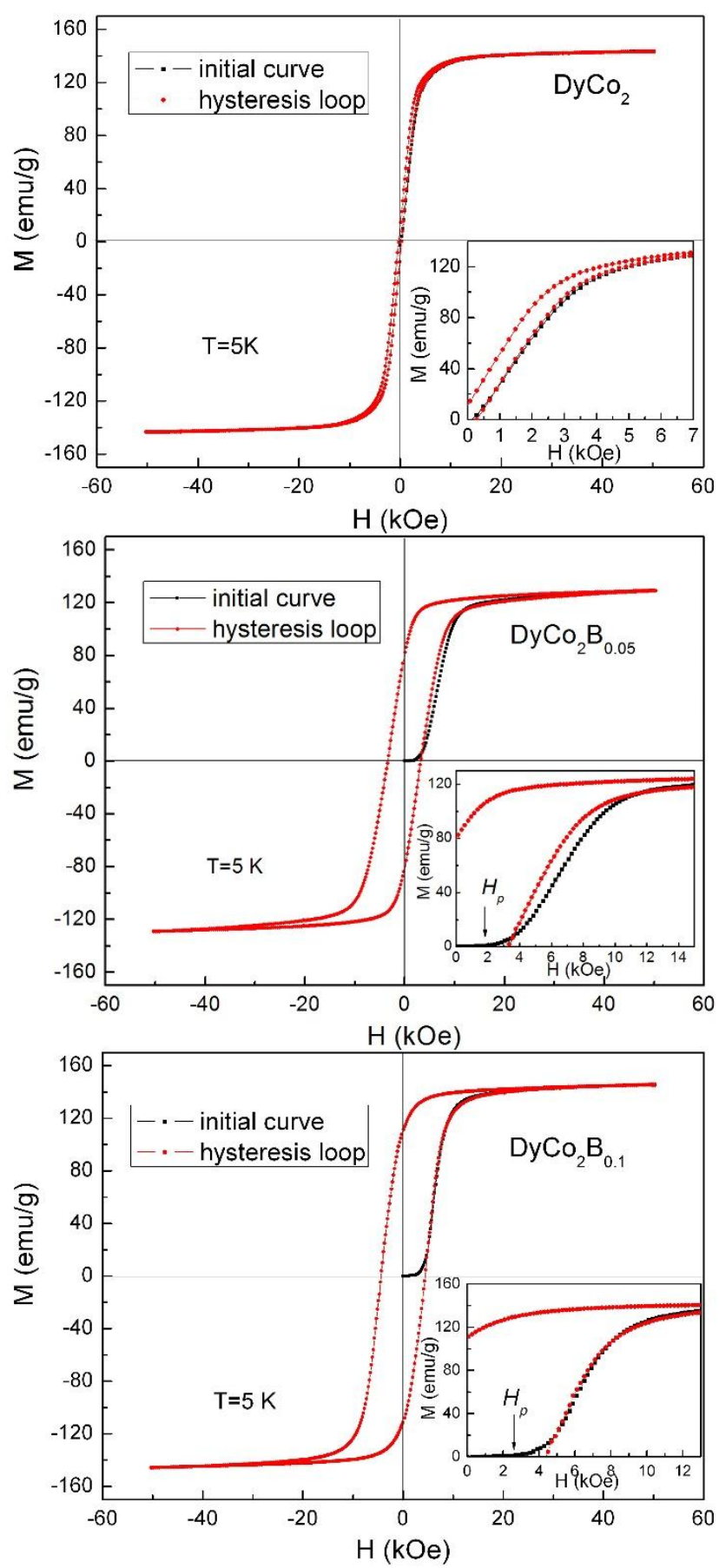

FIG. 5. Hysteresis loops of $\mathrm{DyCo}_{2} \mathrm{~B}_{\mathrm{x}}(\mathrm{x}=0,0.05,0.1)$ measured at $5 \mathrm{~K}$ with magnetic field changing from $-50 \mathrm{kOe}$ to $50 \mathrm{kOe}$. The inset to each graph shows the amplification of the first quadrant.

\section{B. Magnetic relaxation}

To study the time dependence of remanent magnetization of $\mathrm{DyCo}_{2} \mathrm{~B}_{\mathrm{x}}(\mathrm{x}=0,0.05,0.1)$ the isothermal magnetic relaxation measurements were performed and are shown in Fig. 6. 
The samples were first cooled down from $300 \mathrm{~K}$ to $5 \mathrm{~K}$ in magnetic field of $10 \mathrm{kOe}$, and then kept for $1000 \mathrm{~s}$ to stabilize the temperature and magnetization of the sample. After that the magnetic field was turned off and magnetization was measured at $5 \mathrm{~K}$ as a function of time. The magnetic relaxation process can be described by the following expression ${ }^{34}$ :

$$
\mathrm{M}_{\mathrm{r}}=\mathrm{M}_{0}-\mathrm{S}_{\mathrm{v}} \ln \left(\mathrm{t}-\mathrm{t}_{0}\right)
$$

where $M_{r}$ is the remanent magnetization; $M_{0}$ is the initial remanent magnetization; $S_{v}$ is the magnetic viscosity, and $\mathrm{t}$ is time.

The magnetic viscosities $S_{\mathrm{v}}$ of $\mathrm{DyCo}_{2} \mathrm{~B}_{\mathrm{x}}(\mathrm{x}=0,0.05,0.1)$ calculated using equation 2 are $0.49(1)$, 1.85(1) and 1.14(1) emu/g, respectively, as listed in Table 1. The increase of magnetic viscosities of the B doped samples may be due to the larger resistance of domain wall movement caused by the B doping.

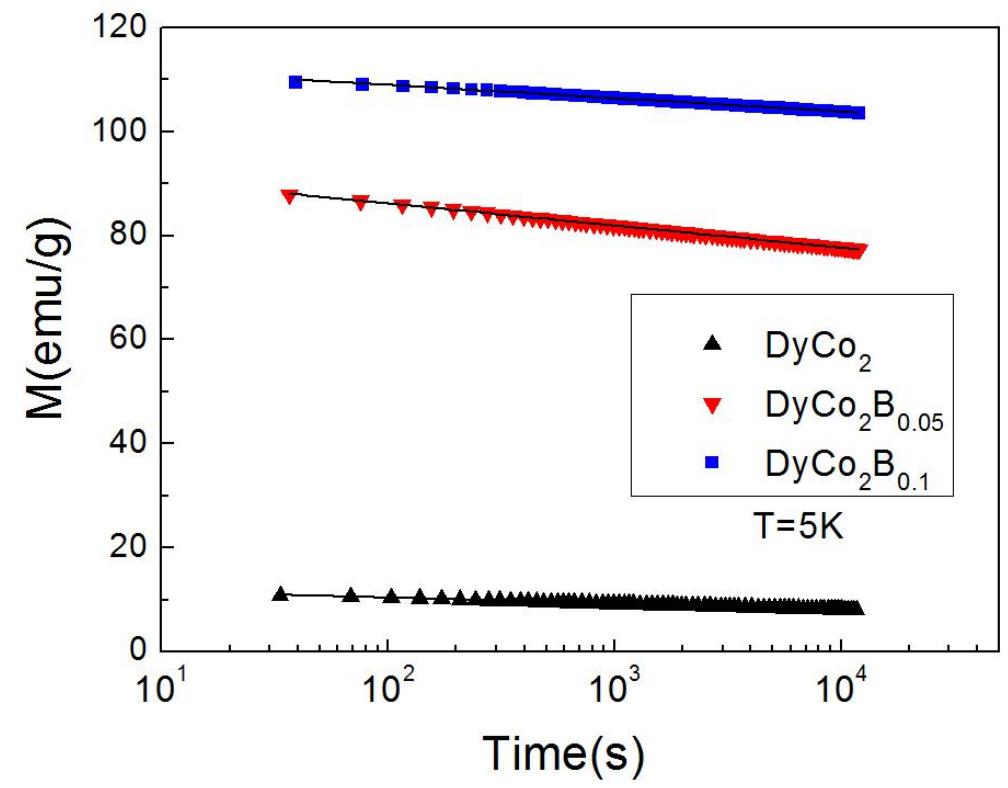

FIG. 6. The remanent magnetization of $\mathrm{DyCo}_{2} \mathrm{~B}_{\mathrm{x}}(\mathrm{x}=0,0.05,0.1)$ magnetized in a field $10 \mathrm{kOe}$ at $5 \mathrm{~K}$. 
Table 1. Crystal and magnetic parameters of $\operatorname{DyCo}_{2} \mathrm{~B}_{\mathrm{x}}(\mathrm{x}=0,0.05,0.1,0.2)$.

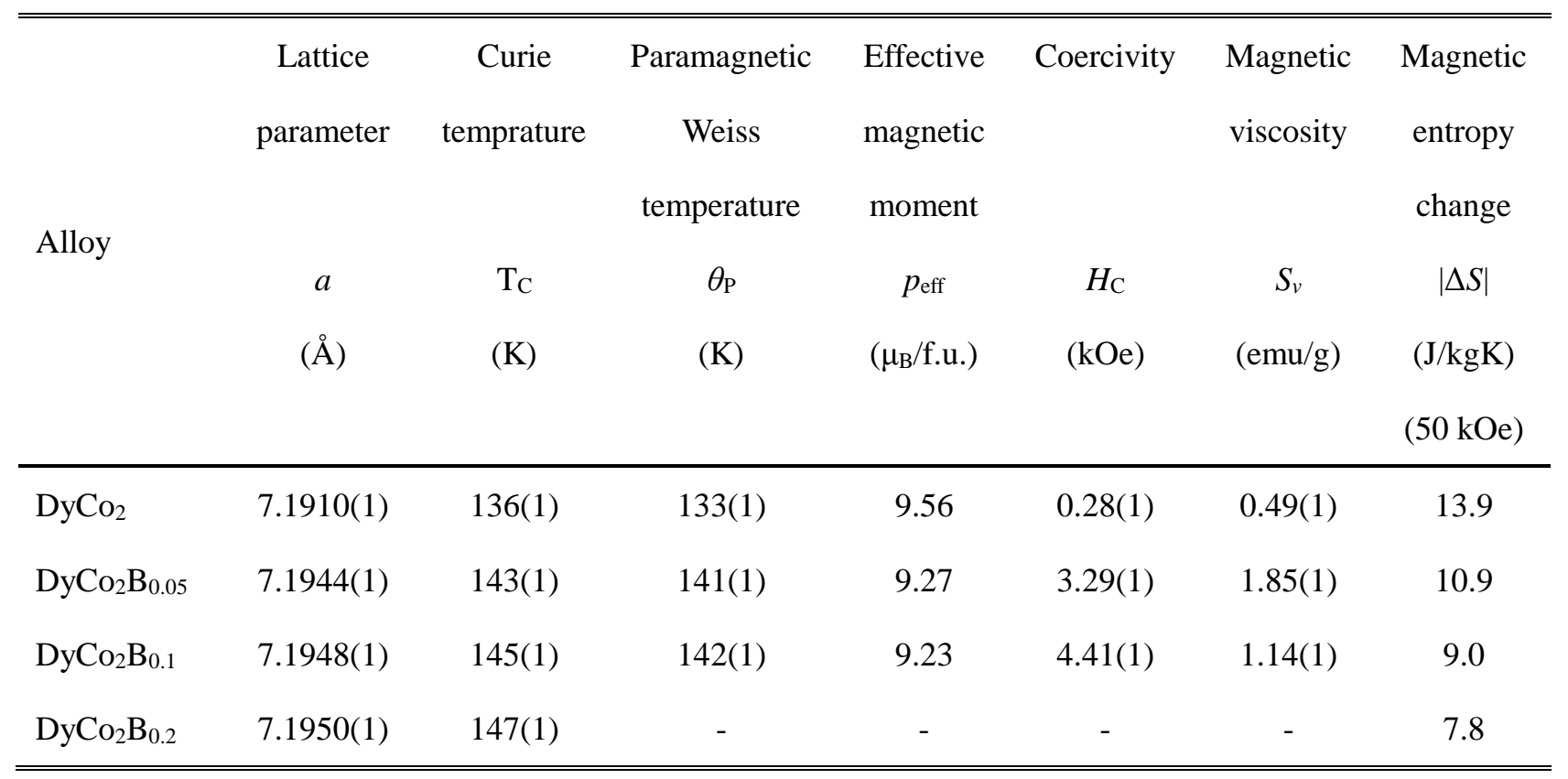

\section{Isothermal magnetization and magnetocaloric effect}

Isothermal magnetization curves of $\mathrm{DyCo}_{2} \mathrm{~B}_{\mathrm{x}}(\mathrm{x}=0,0.05,0.1,0.2)$ are shown in Fig. 7. Both increasing and decreasing field measurements were carried out in the vicinity of $\mathrm{T}_{\mathrm{C}}$. A weak magnetic hysteresis of $\mathrm{DyCo}_{2}$ at $137 \mathrm{~K}$, marked by the arrows in Fig. 7, top left plot, supports the weak first-order nature of magnetic transition. No hysteresis is observed in any of the B doped samples confirming a second order nature of these magnetic transitions. The itinerant electron metamagnetism (IEM) of $\mathrm{DyCo}_{2}$ could be observed just above $\mathrm{T}_{\mathrm{C}} 136 \mathrm{~K}$, but for the $\mathrm{B}$ doped samples the IEM transition is depressed. 

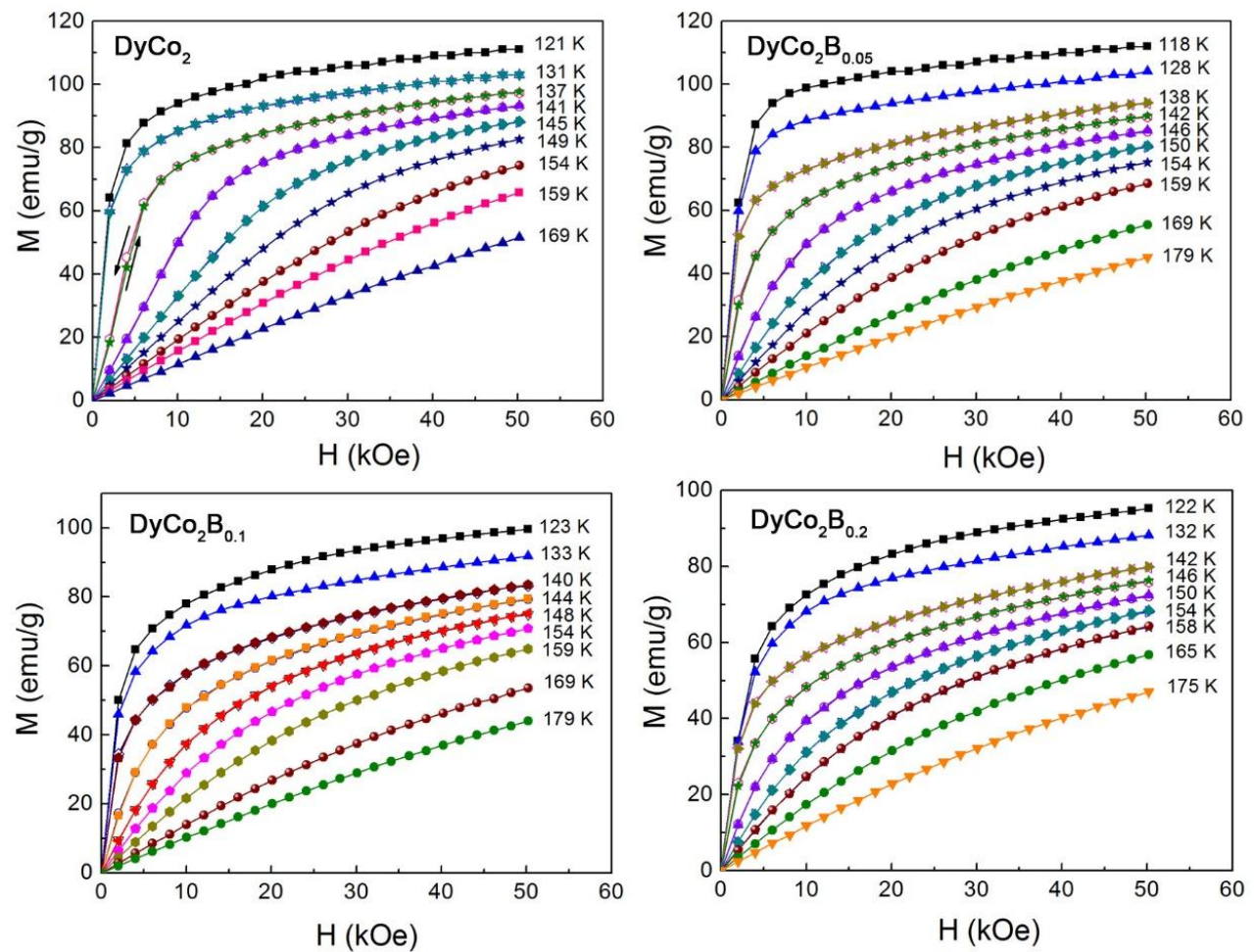

FIG. 7. The magnetization isotherms of $\mathrm{DyCo}_{2} \mathrm{~B}_{\mathrm{x}}(\mathrm{x}=0,0.05,0.1,0.2)$ measured in magnetic fields up to $50 \mathrm{kOe}$.

The Arrott plots of $\mathrm{DyCo}_{2} \mathrm{~B}_{\mathrm{x}}(\mathrm{x}=0,0.05,0.1,0.2)$ obtained just above their respective Curie temperatures are shown in Fig. 8. According to the Banerjee criterion ${ }^{[35]}$, positive slopes of the $\mathrm{M}^{2}$ vs. $\mathrm{M} / \mathrm{H}$ curves of $\mathrm{DyCo}_{2} \mathrm{~B}_{\mathrm{x}}(\mathrm{x}=0.05,0.1,0.2)$ at Curie temperature indicate the second-order magnetic transition. The negative slope of $\mathrm{DyCo}_{2}$ agrees with the first-order nature of magnetic transition. As an alternative to the Banerjee criterion, Bonilla provided a new criterion to distinguish the order of magnetic transitions by rescaling magnetic entropy change in different applied fields ${ }^{[36]}$. If the entropy change curves collapse onto a single one, then the magnetic transition is second order, otherwise it is first order. For $\mathrm{DyCo}_{2} \mathrm{~B}_{\mathrm{x}}(\mathrm{x}=0,0.05,0.1)$ samples, the magnetic entropy change curves are rescaled by two steps: first, normalize each isofield $\Delta \mathrm{S}(\mathrm{T})$ to its maximum value $\Delta \mathrm{S}^{\text {peak }}$, and then rescaling the temperature axis with variable $\theta$, 


$$
\theta=\left\{\begin{aligned}
-\left(\mathrm{T}-\mathrm{T}_{c}\right) /\left(\mathrm{T}_{r 1}-\mathrm{T}_{c}\right), \mathrm{T} \leq \mathrm{T}_{c} \\
\left(\mathrm{~T}-\mathrm{T}_{c}\right) /\left(\mathrm{T}_{r 2}-\mathrm{T}_{c}\right), \mathrm{T}>\mathrm{T}_{c}
\end{aligned}\right.
$$

The reference temperatures $\mathrm{T}_{r 1}$ and $\mathrm{T}_{r 2}$ satisfy $\mathrm{T}_{r 1}<\mathrm{T}_{c}<\mathrm{T}_{r 2}$, and they are determined by the equation 4 , for each curve

$$
\frac{\Delta \mathrm{S}\left(\mathrm{T}_{r 1}\right)}{\Delta \mathrm{S} \text { peak }}=\frac{\Delta \mathrm{S}\left(\mathrm{T}_{r 2}\right)}{\Delta \mathrm{S} \text { peak }}=\frac{1}{2}
$$

As shown in Fig. 8, the rescaled magnetic entropy change curves of $\mathrm{DyCo}_{2} \mathrm{~B}_{\mathrm{x}}(\mathrm{x}=0.05,0.1)$ are collapsed onto single one separately, which confirm their second-order magnetic transition; On the contrary, the scattered curves of $\mathrm{DyCo}_{2}$ demonstrate its first-order magnetic transition.
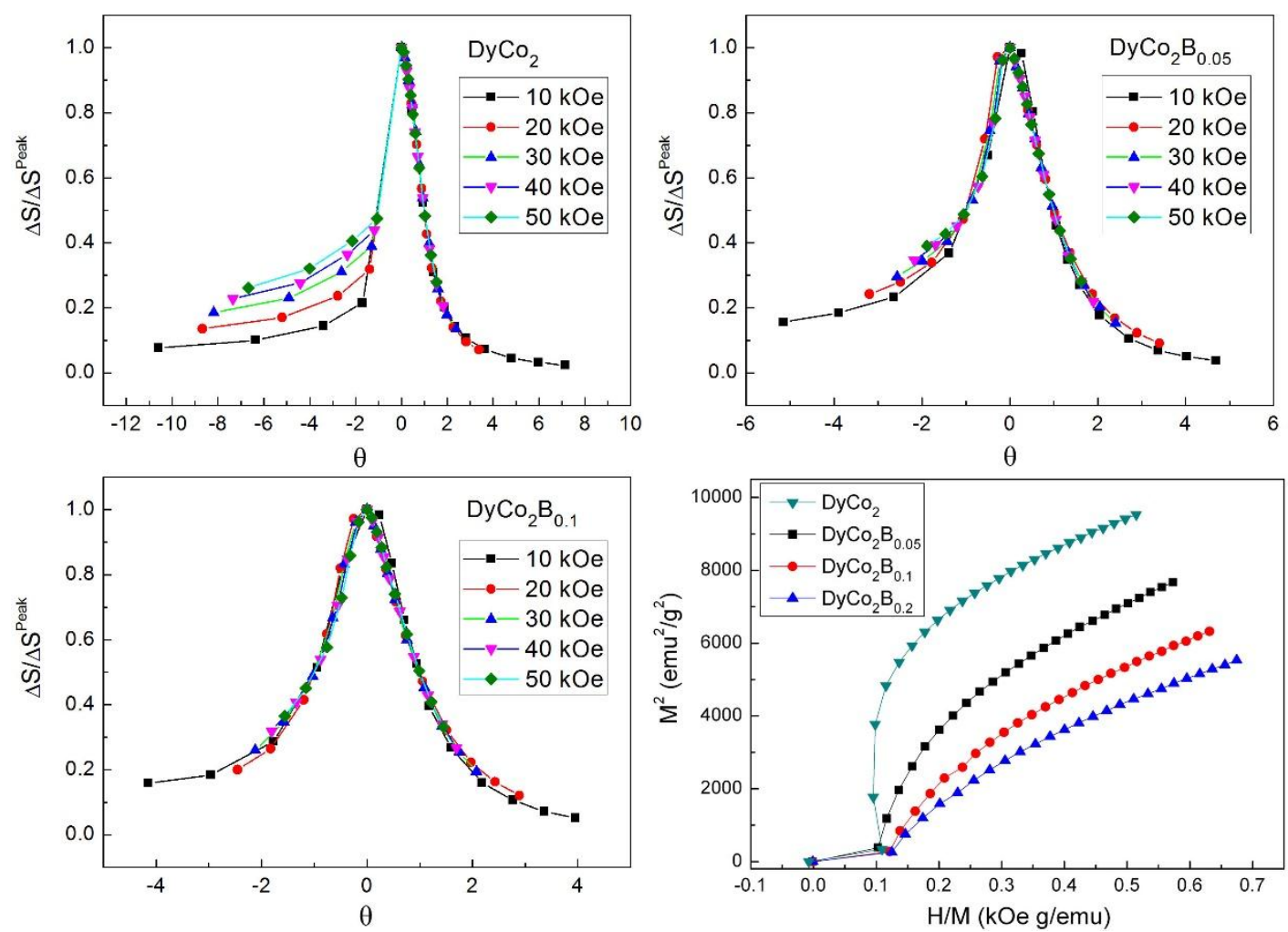

FIG. 8. The scaled entropy change curves and Arrott plots of $\mathrm{DyCo}_{2} \mathrm{~B}_{\mathrm{x}}(\mathrm{x}=0,0.05,0.1,0.2)$.

The plot of magnetic entropy change $(\Delta S)$ versus temperature of $\mathrm{DyCo}_{2} \mathrm{~B}_{\mathrm{x}}(\mathrm{x}=0,0.05,0.1,0.2)$ in the magnetic field of $50 \mathrm{kOe}$, calculated from the isothermal $\mathrm{M}(\mathrm{H})$ data using the Maxwell relation $\Delta S(T, H)=\int_{0}^{H}(\partial M / \partial T)_{H} d H$ is presented in Fig. 9. With increasing of the B concentration, the temperatures of the $\Delta \mathrm{S}(\mathrm{T})$ peaks of $\mathrm{DyCo}_{2} \mathrm{~B}_{\mathrm{x}}$ alloys shift from $136 \mathrm{~K}(\mathrm{x}=0)$ to $147 \mathrm{~K}(\mathrm{x}=0.2)$ 
due to the increase of $\mathrm{T}_{\mathrm{C}}$. But the maximum values decrease from $-13.9 \mathrm{~J} / \mathrm{kg} \cdot \mathrm{K}$ to $-7.8 \mathrm{~J} / \mathrm{kg} \cdot \mathrm{K}$ because the order of the magnetic transition changes from first to second, as shown in Fig. 8 .

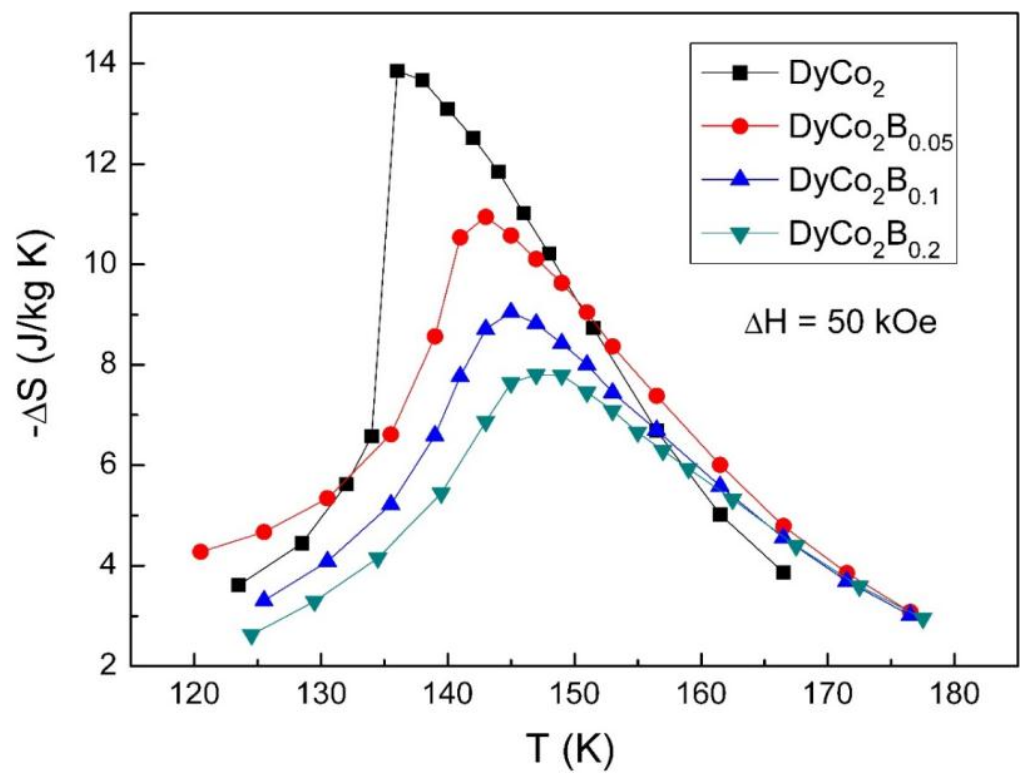

FIG. 9. The entropy change of $\mathrm{DyCo}_{2} \mathrm{~B}_{\mathrm{x}}(\mathrm{x}=0,0.05,0.1,0.2)$ in the magnetic field of $0-50 \mathrm{kOe}$.

The relative cooling power (RCP), which is also an important parameter to evaluate the magnetocaloric materials, is given by the following expression:

$$
R C P=-\Delta S^{\max } \times \delta T_{F W H M}
$$

where $\delta T_{F W H M}$ is the full width at half maximum of $-\Delta \mathrm{S}$ vs. T curve. The RCP values of $\mathrm{DyCo}_{2} \mathrm{~B}_{\mathrm{x}}$ $(\mathrm{x}=0,0.05,0.1,0.2)$ in the magnetic field of $50 \mathrm{kOe}$ are $302,357,310$ and $293 \mathrm{~J} / \mathrm{kg}$. Although the doping of B reduces the maximum entropy change, the $\Delta \mathrm{S}(\mathrm{T})$ peaks become broader. Therefore, the RCP's of $\mathrm{DyCo}_{2} \mathrm{~B}_{\mathrm{x}}$ alloys are almost the same with that of their parent compound.

\section{CONCLUSIONS}

The discrepancy between the ZFC and FCC curves of $\mathrm{DyCo}_{2} \mathrm{~B}_{\mathrm{x}}$ is enhanced in the $\mathrm{B}$ doped samples. 
The Curie temperature of $\mathrm{DyCo}_{2}$ also increases with the $\mathrm{B}$ doping; however, the increase is not linear and when compared with the change in lattice parameters indicates that the $\mathrm{DyCo}_{2} \mathrm{~B}_{\mathrm{x}}$ solid solution is nearly terminated slightly above $\mathrm{x}=0.05$. It is possible that the precipitation of the secondary phase in the grain boundaries also modifies the magnetic properties, such as coercivity. The dissipation of domain wall movement caused by narrow domain wall pinning effect leads to an obvious frequency dependence of the ac magnetic susceptibility in DyCo2. However, this phenomenon is masked in the B doped samples, because the domain walls are further stabilized by B doping at low temperature where narrow domain wall pinning effect is strong, while at higher temperatures the increase in thermal energy overcomes the kinetic effects associated with pinning. The coercivity and viscosity that are related with the mobility of the domain walls are increased by the B doping. The magnetic transition nature is changed from first-order to second-order because of the B doping, which leads to the decrease of the maximum magnetic entropy change values. At the same time the RCP's of $\mathrm{DyCo}_{2} \mathrm{~B}_{\mathrm{x}}$ for $\mathrm{x}=0.1$ and 0.2 are almost the same (within $3 \%$ ) with that of the parent compound $\mathrm{DyCo}_{2}$, while that for $\mathrm{x}=0.05$ is about $18 \%$ larger.

\section{ACKNOWLEDGEMENT}

The Ames Laboratory is operated for the U.S. Department of Energy by Iowa State University of Science and Technology. This work was supported by the Department of Energy, Office of Basic Energy Sciences, Materials Sciences Division under Contract No. DE-AC02-07CH11358. C. L. W. and Y. L. acknowledge the National Science Foundation of China, the National High Technology Research and Development Program of China, the National Basic Research Program of China, and the funding from China Scholarship Council for support of C. L. W. stay at the Ames Laboratory. 


\section{References}

$1 \quad$ R. Levitin, A.S. Markosyan, J. Magn. Magn. Mater. 84 (1990) 247.

2 N. K. Singh, K. G. Suresh, A. K. Nigam, S. K. Malik, A. A. Coelho, and S. Gama, J. Magn. Magn. Mater. 317 (2007) 68.

N. H. Duc, D. T. Kim Anh, and P. E. Brommer, Physica B: Condens. Matter 319 (2002) 1.

E. Gratz, A.S. Markosyan J. Phys.: Condensed Matter 13 (2001) R385.

A. Kowalczyk, J. Baszyński, A. Szajek, J. Kováč, and I. Škorvánek, J. Magn. Magn. Mater. 152 (1996) L279.

N. K. Singh, K. G. Suresh, and A. K. Nigam, Solid State Commun. 127 (2003) 373.

A. Del Moral, and D. Melville, J. Phys. F. Metal Physics 5 (1975) 1767.

L. C. Sampaio and S. F. da Cunha, J. Magn. Magn. Mater. 99 (1991) 145.

V. K. Pecharsky and K. A. Gschneidner, Phys. Rev. Lett. 78 (1997) 4494.

F. X. Hu, B. G. Shen, J. R. Sun, Z. H. Cheng, G. H. Rao, and X. X. Zhang, Appl. Phys. Lett. 78 (2001) 3675.

O. Tegus, E. Bruck, K. H. J. Buschow, and F. R. de Boer, Nature 415 (2002) 150.

H. Wada and Y. Tanabe, Appl. Phys. Lett. 79 (2001) 3302.

F. X. Hu, B. G. Shen, and J. R. Sun, Appl. Phys. Lett. 76 (2000) 3460.

T. Krenke, E. Duman, M. Acet, E. F. Wassermann, X. Moya, L. Manosa, and A. Planes, Nat. Mater. 4 (2005) 450.

A. Fujita, S. Fujieda, Y. Hasegawa, and K. Fukamichi, Phys. Rev. B Phys. 67 (2003) 104416.

B. G. Shen, J. R. Sun, F. X. Hu, H. W. Zhang, and Z. H. Cheng, Adv. Mater. 21 (2009) 4545.

D. Wang, H. Liu, S. Tang, S. Yang, S. Huang, and Y. Du, Phys. Lett. A 297 (2002) 247. 
N.A. de Olivera, J. Magn. Magn. Mater. 320 (2008) e150.

W. Ao, J. Li, F. Liu, and Y. Jian, Solid State Commun. 141 (2007) 219.

Z. Han, Z. Hua, D. Wang, C. Zhang, B. Gu, and Y. Du, J. Magn. Magn. Mater. 302 (2006) 109.

A. Kowalczyk, A. Szajek, J. Baszyński, J. Kováč, and G. Chełkowska, J. Magn. Magn. Mater. 166 (1997) 237.

N. K. Singh, P. Kumar, K. G. Suresh, A. K. Nigam, A. A. Coelho, and S. Gama, J. Phys.: Condens. Matter 19 (2007) 036213.

H. W. Li, K. Ishikawa, and K. Aoki, Mater. Sci. Forum. 475 (2005) 2469.

Materials Preparation Center, The Ames Laboratory of U.S. Department of Energy, Ames, IA, USA, www.mpc.ameslab.gov.

B.A. Hunter, Rietica - A Visual Rietveld Program, International Union of Crys-tallography Commission on Powder Diffraction Newsletter, No. 20 (Summer, 1998), http://www.rietica.org.

J. A. Chelvane and G. Markandeyulu, J. Magn. Magn. Mater. 281 (2004) 1.

J. J. Didisheim, K. Yvon, P. Fischer, and D. Shaltiel, J. Less Com. Met. 73 (1980) 355.

J. L. Wang, C. Marquina, M. R. Ibarra, and G. H. Wu, Phys. Rev. B 73 (2006) 094436.

M. Khan, Y. Mudryk, D. Paudyal, K. Gschneidner, and V. Pecharsky, Phys. Rev. B 82 (2010) 064421.

J. Herrero-Albillos, F. Bartolomé, L. M. García, A. T. Young, T. Funk, J. Campo, and G. J. Cuello, Phys. Rev. B 76 (2007) 094409.

D.K. Zhang, J.L. Zhao, J. Shen, H.G. Zhang, M. Yue, J. Appl. Phys. 115 (2014) 183908.

K. Binder and A. P. Young, Rev. Mod. Phys. 58 (1986) 801. 

Appl. Phys. 95 (2004) 6678.

D. Pajic, K. Zadro, R. Ristic, I. Zivkovic, Z. Skoko, and E. Babic, J. Phys.: Condens. Matter 19 (2007) 296207. S.K. Banerjee, Physics Letters, 12 (1964) 16-17. J. Appl. Phys. 107 (2010) 09E131. 